\section{The genetics workforce and workload}

\section{To the Editor:}

With the field of genetics rapidly advancing, the capacity of the workforce of genetics specialists will be critical to the implementation of new genetic diagnostic tools and therapies in the coming years. Although all health professionals must achieve a minimum level of competency in genetics to appropriately apply genetics to the practice of medicine, genetics specialists remain an important group of experts in filling the roles of teachers, research scientists, laboratory directors, counselors, nurses, social workers, and clinical specialists. A 1994 Institute of Medicine report predicted that while the traditional role of genetics specialists will change, they will continue to fill three increasingly important roles: (1) providing genetic testing and counseling for complex genetic disorders, (2) training and educating other health professionals in genetics, and (3) providing specialized genetic services. ${ }^{1}$ In light of the expansion of genetics in medicine and research, we recommend that a comprehensive workforce assessment be conducted to ascertain the capabilities of genetics specialists to fulfill their multiple responsibilities now and in the future.

We define genetics specialists here as individuals who are board-certified by the American Board of Medical Genetics (ABMG) or the American Board of Genetic Counseling (ABGC). We recognize that there are many other health professionals with substantial expertise and experience in genetics who are not certified by these two boards, but because of the lack of published relevant data, we have limited our comments to those who are board-certified in genetics or genetic counseling.

In 1980, ABMG was established to accredit training programs in genetics and to certify individuals who provide medical genetics services. In 1991, ABMG was accepted into the American Board of Medical Specialties (ABMS) as its 24th member board to approve the certification of professionals with doctoral degrees (MD or PhD). Currently, there are 45 accredited clinical genetics residency programs ${ }^{2}$ and 43 laboratory training programs in the United States. ${ }^{3}$

ABMG certification in genetics is offered in five areas: clinical genetics (MD or DO), medical genetics ( $\mathrm{PhD}$ only), clinical cytogenetics (MD, DO, and/or $\mathrm{PhD}$ ), clinical biochemical genetics ( $\mathrm{MD}, \mathrm{DO}$, and/or $\mathrm{PhD})$, and clinical molecular genetics (MD, DO, and/or PhD). A dual certificate in clinical biochemical and molecular genetics was offered once in 1990. A joint subspecialty certification in molecular genetic pathology was recently established by ABMG and the American Board of Pathology and approved by ABMS in 1999. The first examination in molecular genetic pathology (MD only) was offered in fall 2001.

Because of ABMS stipulations that member boards may not certify master's-level candidates, genetic counselors could no longer be part of ABMG upon its acceptance into ABMS. Therefore, ABGC was incorporated in 1991 to accredit training programs and certify genetic counselors. Currently, there are
25 genetic counseling programs in the United States, ${ }^{4}$ graduating approximately 120 master's-trained professionals annually. ${ }^{5}$

Certifying examinations in genetics are offered every 3 years. As of the last examination (1999), a total of 2191 certificates had been awarded to 1847 individuals (344 are certified in more than one specialty). ${ }^{6}$ Approximately 900 are certified as MD clinical geneticists practicing in the United States. Between 1993 and 1996, 21\% fewer certificates were awarded overall, and between 1996 and 1999, 32\% fewer certificates were awarded. In 1993, certifying examinations in clinical molecular genetics were offered for the first time, accounting for the large number of individuals certified in that specialty that year (therefore, skewing the apparent decrease in 1996). In 1999, the number of certificates awarded was down in each of the five specialties, the most precipitous drop noted in MD clinical genetics (28\%). However, among all the genetics specialties, clinical genetics (MD) remains the largest group (1006 total), followed by clinical cytogenetics (522) and clinical molecular genetics (284). The number of individuals approved for the 2002 board examinations shows a decline from 1999 in all of the genetics specialties (personal communication, Ms. Sharon Robinson, ABMG/ABGC Administrator, August 9, 2002).

In contrast to the declining numbers of individuals receiving board certification in clinical and medical genetics, the number of certified genetic counselors has increased, almost doubling between 1993 and 1999. Between 1993 and 1996, 30\% more certificates were awarded and 24\% more were awarded between 1996 and 1999. As of 1999, 1410 individuals were certified in genetic counseling. If the total number of individuals certified as specialists in clinical and medical genetics and genetic counseling are combined, the increase in genetic counselors is canceled out by the decrease in clinical and medical geneticists, resulting in a flat number of certificates $(\sim 516)$ issued in each 3-year cycle since 1993.

Because most genetics specialists work in academic settings, they serve the traditional tripartite mission of an academic institution-clinical service, teaching, and research.

In addition to serving as a genetics referral resource to a large number of primary care and other specialty physicians, clinical geneticists may also serve as primary care providers. Most MD clinical geneticists are certified in another medical specialty in addition to genetics, most frequently pediatrics $(63 \%)$ followed by obstetrics and gynecology (13\%) and internal medicine $(9 \%)$. About $10 \%$ are certified in genetics only.

Laboratory geneticists play critical roles in basic research and development as well as in the translation, implementation, and provision of genetic tests and services. Genetic testing is expanding rapidly with the near completion of the human genome sequence and new identification of genes that are responsible for, or predictive of, increased disease risk. While the number of genetic tests conducted annually in the United States is difficult to estimate (the most recent figures show an increase from almost 100,000 tests performed in 1994 to more than 175,000 in $1996^{7}$ ), the number of clinically available ge- 
netic tests has increased from 360 in 1999 to 548 in $2002 .{ }^{8}$ In recognition of the rapid increase in the numbers and types of genetic tests and lack of current oversight, state and federal regulations governing genetic testing laboratories are being written and laboratory standards and guidelines are being strengthened. The US Department of Health and Human Services is planning to issue a proposed rule augmenting the Clinical Laboratories Improvement Amendments regulations to establish a genetic testing specialty. ${ }^{9}$ This increase in testing and laboratory regulations is likely to result in an increased clinical testing workload for certified geneticists.

The role of genetics specialists as basic science and clinical instructors is critical to the transmission of genetics information to students, residents, and practicing health professionals of many disciplines as well as the general public. For genetics specialists at academic medical centers, teaching responsibilities can be varied and extensive throughout undergraduate and graduate medical education. In addition, not all medical and graduate schools have a department or division of genetics, leaving genetics specialists employed in other traditional medical departments to provide genetics instruction on their own. The teaching workload often involves lecturing, not only in the basic medical school curriculum, but also in other basic science courses in dentistry, nursing, allied health, pharmacy, social work, and graduate schools. While some elective genetics rotations occur in the third and fourth years of medical school and in residency training, the inclusion of genetics content in primary care training programs is sporadic. Third- and fourthyear medical students as well as some $\mathrm{PhD}$ students may rotate through pediatric or prenatal clinics with a genetics specialist.

Academic-based geneticists are also responsible for the training of master's- and doctoral-level students in graduate programs in human genetics and molecular biology, including lecturing in didactic coursework and mentoring trainees in their thesis and dissertation research. Certified genetic counselors direct nearly all genetic counseling training programs, and clinical geneticists are an essential faculty component required of accredited programs. Genetic counselors at academic medical centers with genetic counseling programs often lecture or teach courses, serve as mentors, and oversee student clinical rotations. Faculty and instructor-level genetic counselors also lecture on genetics in other professional schools including nursing, medicine, social work, and allied health.

Investigational research activities remain a primary obligation of many academic-based geneticists. Basic and translational research efforts are extremely vital to the continued rapid growth of knowledge in genetics and its clinical application. In addition to the income derived from clinical services of genetics specialists, many academic institutions rely on genetics specialists to also attract research grant dollars.

In light of the growing importance of genetics specialists to provide comprehensive genetics services, educate, and conduct research, it is essential to evaluate the ability of the genetics workforce to carry out its many responsibilities. No thorough assessment has yet been carried out regarding the composition, number, training, and professional practices of genetics specialists or the obstacles they may encounter in providing their services. Two studies have evaluated workforce issues, but only as they relate to genetic counselors and pediatric genetic specialists. ${ }^{5,10}$ The workforce study of genetic counselors concluded that the number of genetic counselor training programs should be maintained at current levels, but that the job market and rate of new clinical applications of genetic testing should be monitored to detect changes in demand.

Assessing the workload issues faced by genetics specialists is a critical step in evaluating the capabilities of the genetics workforce. Because of the increasing importance and presence of genetics in medicine and the lack of growth of the genetics workforce, action may be needed to ensure that an adequate number of genetics health professionals are maintained to usher in this new era. It will be important to determine the amount of time spent on the various activities of genetics specialists—-for example, do geneticists spend the majority of their time seeing patients or do they spend more time on teaching and research? Furthermore, it would be appropriate to reflect on the multiple roles genetics specialists currently fill as briefly described here and how they may evolve in the future.

The decline of board-certified MD genetics specialists certainly warrants further investigation. The number of employment opportunities, inadequate institutional support of genetics as a priority, inadequate reimbursement for genetics specialists, lack of genetics in undergraduate health curricula and postgraduate training, and anecdotal evidence of negative perceptions of the field of genetics as a specialty by new graduates may provide reasons for the decline. In any case, the expected increases in demand for genetics services without a concomitant increase in supply will inevitably place strains on the existing pool of geneticists. With a greater clinical load, geneticists may have less time per patient and less time for teaching and research. Transferring some of these responsibilities to other, less-specialized (and perhaps less qualified) health care providers will be the necessary result. It will be important to determine how many certified genetics specialists are actually practicing and to project how many will be retiring in the next 5 to 10 years.

A study funded by the Health Resources and Services Administration will examine the clinical practices of $\mathrm{MD}$ and $\mathrm{PhD}$ board-certified geneticists and collect data through a survey regarding patient volume, referrals, and professional satisfaction. However, this study was not designed to address the multiple roles of genetics specialists described here. It will also be important to assess not only the number of people providing clinical and laboratory genetics services, but those teaching genetics and conducting genetics research who are not boardcertified genetics specialists. While the number of people applying for board certification is clearly declining, the number of individuals providing genetics services overall may not be.

In addition, two other important considerations are the diversity and distribution of the genetics workforce. Workforce diversity is a universally important element in today's health care system. A culturally diverse and competent workforce helps build patient-provider relationships based on confi- 
dence and trust to serve the multicultural population health care needs of the people living in the United States. Data collected from the studies of pediatrics genetics specialists and genetic counselors show that the majority are Caucasian., ${ }^{5,10}$ Another important element is the location of genetics specialists. Because many genetics specialists are concentrated in academic medical centers and research institutions in major cities and suburban areas, patients and consumers residing in smaller cities or rural areas will have limited access to genetics specialists or comprehensive genetic care.

The future of genetics in science and medicine is bound to be exciting and revolutionary. However, the fate of genetics specialists and their future role(s) remain to be determined. A comprehensive workforce assessment will allow the genetics community to take a closer look at themselves and, it is hoped, provide an opportunity to shape their fate and the future of the specialty that will benefit all involved.

Disclaimer: Data cited in this letter were collected in support of a comprehensive analysis of genetics issues by the HHS Secretary's Advisory Committee on Genetic Testing. However, the views expressed here are solely those of the authors and do not reflect the views or position of SACGT, ASHG, or The Center for the Advancement of Genomics.

Susanne B. Haga, PhD* Office of Biotechnology Activities National Institutes of Health Bethesda, Maryland

Joann A. Boughman, PhD American Society of Human Genetics Bethesda, Maryland

\section{References}

1. Andrews LB, Holtzman NA, Motulsky A, editors. Assessing genetic risks: implications for health and social policy. Washington, DC: National Academy Press, 1994.

2. American Council of Graduate Medical Education. Reports: programs by specialty (medical genetics), 2001. http://www.acgme.org/adspublic/reports/program_specialty_list.asp.

3. American Board of Medical Genetics and American Society of Human Genetics. Guide to North American graduate and postgraduate training programs in human genetics, 2001. http://www.abmg.org/genetics/ashg/tpguide/tpg-menu.htm.

4. American Board of Genetic Counseling. Genetic counseling training programs accredited by the ABGC, 2001. http://www.abgc.net/genetics/abgc.tr-prog1.html.

5. Cooksey JA. The genetic counselor workforce: training programs, professional practice, and issues affecting supply and demand. Chicago: Center for Health Workforce Studies, February 2000.

6. American Board of Medical Genetics. Numbers of certified specialists in genetics, 2001. http://www.faseb.org/genetics/abmg/stats-allyears.htm.

7. McGovern MM, Benach MO, Wallenstein S, Desnick RJ, Keenlyside R. Quality assurance in molecular genetic testing laboratories. JAMA 1999;281:835-840.

8. GeneTests. Summary reports requested by the Secretary's Advisory Committee on Genetic Testing (11/05/99 and 04/01/02). http://www.genetests.org.

9. Department of Health and Human Services, Centers for Disease Control and Prevention. Notice of intent: genetic testing under the clinical laboratory improvement amendments. Federal Register 2000;65(87):25928-25934.

10. Future of Pediatric Education II: a project of the pediatrics community. Summary of survey findings: clinical genetics. American Academy of Pediatrics, 1998.

\section{Tetrahydrobiopterin control in phenylketonuria}

To the Editor:

In patients with phenylketonuria (PKU), blood phenylalanine concentration during childhood is the major determinant of cognitive outcome. Adolescents and young adults generally do not comply with recommendations for the monitoring and control of phenylalanine concentrations, ${ }^{1}$ and two thirds of pregnant women in the United States did not follow the diet before becoming pregnant. ${ }^{2}$

Recently it has been shown that some patients with PKU respond to the loading test with 6R-tetrahydrobiopterin $\left(\mathrm{BH}_{4}\right)$ by lowering plasma phenylalanine concentrations and that these patients can be treated with $\mathrm{BH}_{4} \cdot{ }^{3} \mathrm{BH}_{4}$ is the natural cofactor of aromatic amino acid hydroxylases, and particular mutations in the phenylalanine hydroxylase gene may affect enzyme stability or its affinity for the $\mathrm{BH}_{4}$. We recently found that more than $60 \%$ of patients with plasma phenylalanine concentrations between 400 and $800 \mu \mathrm{mol} / \mathrm{L}$ respond to $\mathrm{BH}_{4}$ challenge and that the best results can be obtained with doses of $20 \mathrm{mg} / \mathrm{kg}$ body weight (Fig. 1). ${ }^{4}$ Oral supplementation with 10 $\mathrm{mg} \mathrm{BH}_{4} / \mathrm{kg}$ reduced plasma phenylalanine concentrations below $360 \mu \mathrm{mol} / \mathrm{L}$ in most patients with mild forms of phenylalanine hydroxylase deficiency (Phe $<1200 \mu \mathrm{mol} / \mathrm{L}$ ) and increased phenylalanine tolerance in some patients with classical PKU $($ Phe $>1200 \mu \mathrm{mol} / \mathrm{L})$.

Our findings show the importance of the $\mathrm{BH}_{4}$ loading test in the diagnosis of $\mathrm{BH}_{4}$-responsive variants and open new prospects for the treatment of PKU and mild hyperphenylalaninemia. Replacement of the low-phenylalanine diet with the commercially available $\mathrm{BH}_{4}$ (Schircks Laboratories, Jona, Switzerland) may significantly improve compliance in adolescents and young adults

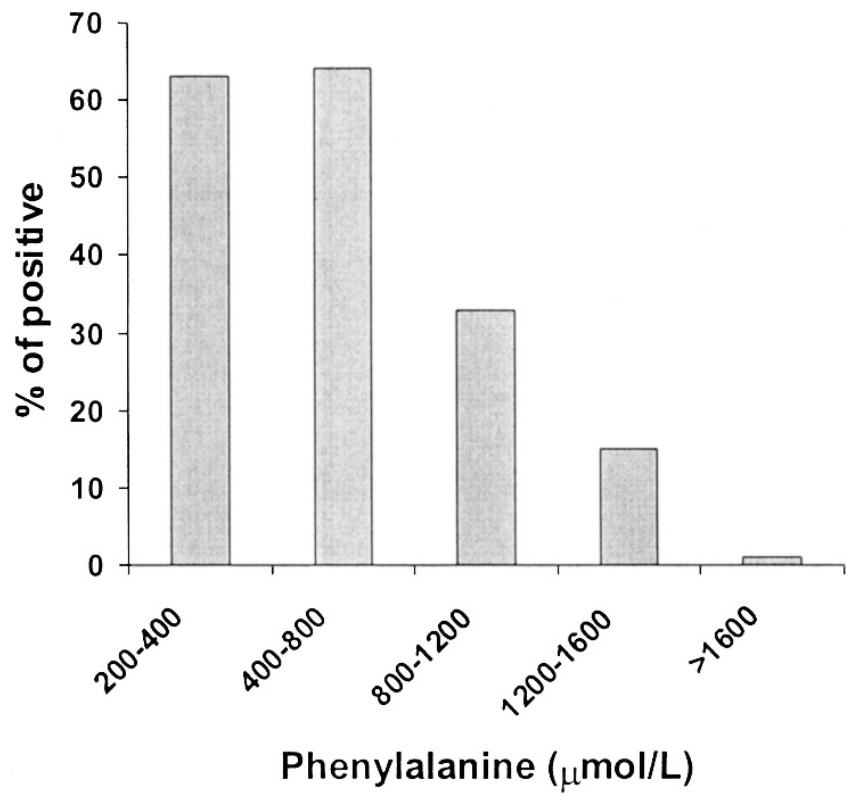

Fig. 1 Percentage of patients with different degrees of hyperphenylalaninemia who responded to $6 \mathrm{R}$-tetrahydrobiopterin challenge $(20 \mathrm{mg} / \mathrm{kg}$ body weight) by lowering plasma phenylalanine levels by at least $30 \%$ within 8 hours. 